\title{
ARTICLE
}

Molecular Diagnostics

\section{MicroRNA-196a is regulated by ER and is a prognostic biomarker in ER+ breast cancer}

\author{
Michael J. G. Milevskiy ${ }^{1,6}$, Udai Gujral ${ }^{1}$, Carolina Del Lama Marques ${ }^{1}$, Andrew Stone ${ }^{2}$, Korinne Northwood ${ }^{1,3}$, Lez J. Burke ${ }^{1}$,
} Julia M. W. Gee ${ }^{4}$, Kenneth Nephew ${ }^{5}$, Susan Clark $^{2}$ and Melissa A. Brown ${ }^{1}$

BACKGROUND: MicroRNAs are potent post-transcriptional regulators involved in all hallmarks of cancer. Mir-196a is transcribed from two loci and has been implicated in a wide range of developmental and pathogenic processes, with targets including Hox, Fox, Cdk inhibitors and annexins. Genetic variants and altered expression of MIR196A are associated with risk and progression of multiple cancers including breast cancer, however little is known about the regulation of the genes encoding this miRNA, nor the impact of variants therein.

METHODS: Genomic data and chromatin interaction analysis were used to discover functional promoter and enhancer elements for MIR196A. Expression data were used to associate MIR196A with mechanisms of resistance, breast cancer subtypes and prognosis. RESULTS: Here we demonstrate that MIR196A displays complex and dynamic expression patterns, in part controlled by long-range transcriptional regulation between promoter and enhancer elements bound by ERa. Expression of this miRNA is significantly increased in drug-resistant models of hormone-receptor positive disease. The expression of MIR196A also proves to be a robust prognostic factor for patients with advanced and post-menopausal ER+ disease.

CONCLUSION: This work sheds light on the normal and abnormal regulation of MIR196A and provides a novel stratification method for therapeutically resistant breast cancer.

British Journal of Cancer (2019) 120:621-632; https://doi.org/10.1038/s41416-019-0395-8

\section{BACKGROUND}

MicroRNAs are short non-coding RNAs that post-transcriptionally regulate gene expression. ${ }^{1}$ MicroRNAs have been implicated in many diseases, including rare inherited syndromes, arising from germline mutations in MiRNA genes, and several cancers types. ${ }^{2}$ Research into the biology and pathology of these molecules has led to the identification of clinically useful genetic and epigenetic biomarkers and more recently novel therapeutic agents. $^{3}$ These therapeutic agents are based on antagomiR technology, synthetic RNA molecules that bind miRNA targets, and have shown promise in the control of disease symptoms and progression.

MicroRNA-196A (mature RNA MIR196A, non-human miR196a) is transcribed from two genomic loci, HOXC (Chr12 in humans, gene MIR196A2) and HOXB (Chr17 in humans, gene MIR196A1), both situated upstream of $H O X 9$, respectively. ${ }^{4}$ The precursor transcript expressed from MIR196A2 (pre-MIR196A2) produces two mature miRNA molecules, miR-196a-5p (herein referred to as MIR196A, miR196a non-human) and miR-196a-3p, whilst the HOXB precursor gene MIR196A1 (pre-MIR196A1) also encodes miR-196a-5p but a different $3^{\prime}$ miRNA, miR-196a-1-3p. Early studies into the function of miR196a in mice and chicken, demonstrated a requirement for miR196a expression to suppress
Hoxb8 RNA, essentially controlling its spatiotemporal pattern along the anterior-posterior axis. ${ }^{5-8}$

MIR196A been implicated in a range of cancers, primarily as an oncogene. For example, MIR196A is overexpressed in breast tumours relative to normal breast tissue, ${ }^{9}$ and additionally a single nucleotide polymorphism (SNP, rs11614913, C>T) within the MIR196A2 gene is associated with a decreased risk of breast cancer. ${ }^{10}$ The decrease in risk from rs11614913 was found to be associated with a decrease in processing of the precursor transcript to mature miRNA, resulting in less MIR196A expression and highly suggestive of an oncogenic role in breast cancer. MIR196A has also been shown to target the $3^{\prime}$ UTR of Annexin-1 (ANXA1), an important mediator of apoptosis, ${ }^{11}$ in response to the pro-angiogenic vascular endothelium growth factor (VEGF), leading to alterations in angiogenesis, a hallmark of tumourigenesis. ${ }^{12}$ A separate study demonstrated that MIR196A could increase growth, migration and invasion of a non-small-cell lung cancer cell line through direct targeting of HOXA5. ${ }^{13}$ Two studies have recently shown that MIR196A can directly influence the cell cycle by targeting $\mathrm{p} 27 / \mathrm{Kip} 1$, an inhibitor of cell cycle progression, to dramatically increase growth and pro-oncogenic features of cancer cell lines. ${ }^{14,15}$ Despite the clear importance on MIR196A in cancer, its transcriptional regulation remains poorly understood.

\footnotetext{
${ }^{1}$ School of Chemistry and Molecular Biosciences, University of Queensland, St Lucia, QLD, Australia; ${ }^{2}$ Division of Genomics and Epigenetics, Epigenetics Research Laboratory,

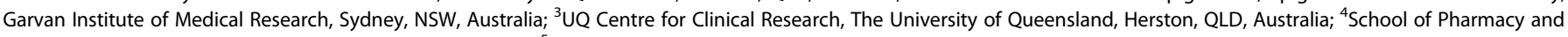
Pharmaceutical Sciences, Cardiff University, Cardiff, UK and ${ }^{5}$ School of Medicine, Indiana University, Bloomington, IN, USA Correspondence: Michael J. G. Milevskiy (milevskiy.m@wehi.edu.au)

${ }^{6}$ Present address: ACRF Stem Cells and Cancer, The Walter and Eliza Hall Institute of Medical Research, Parkville, VIC, Australia
}

Received: 24 May 2018 Revised: 16 December 2018 Accepted: 15 January 2019

Published online: 20 February 2019 
Transcriptional regulation is a complex multi-faceted biological process that is significantly altered in cancer. MicroRNA genes are regulated transcriptionally in a similar manner to protein coding and long non-coding RNA genes. Promoters mostly lie upstream (within $10 \mathrm{~kb}$ of the mature miRNA), contain a CpG island and in an active state when the miRNAs are transcribed by RNA Pol II are enriched for H3K4me3 and lack H3K27me3 similar to protein coding genes. ${ }^{16,17}$ Taken together, these data indicate that potential promoters for miRNAs can be identified in a similar manner to methods for protein coding genes. Several instances of miRNA regulation by enhancers have been described, but this area is very much in its infancy. ${ }^{18,19}$

In this study, we aimed to characterise the expression landscape of MIR196A including factors regulating its expression and explore potential roles of regulatory elements and factors in breast cancer prognostication.

\section{MATERIAL AND METHODS}

\section{Cell culture}

MCF7 cells, for the development of endocrine resistance sub-lines were obtained from AstraZeneca. MCF7, Tamoxifen-resistant (TAMR), Fulvestrant-resistant (FASR), and oestrogen-deprived (MCF7x) cells were cultured as described. ${ }^{20-22}$ All cell lines were cultured for less than 6 months after authentication by shorttandem repeat (STR) profiling (Cell Bank, Australia). MCF7 cells were cultured in RPMI (ThermoFisher, 11875-093) supplemented with $5 \%$ foetal calf serum (FCS, ThermoFisher, 1600-044). TAMR, FASR and MCF7x lines were cultured in phenol-red free RPMI (ThermoFisher, 11835-030) supplemented with 5\% charcoal stripped FCS (Sigma-Aldrich, F6765), additionally TAMR cells were maintained in 4-Hydroxytamoxifen (Sigma H7904, 10-7 M) and FASR cells in Fulvestrant (Herceptin ${ }^{\oplus}$, Genentech, $10^{-7} \mathrm{M}$ ).

\section{Cloning and reporter assays}

All PCR products for luciferase reporter assays were ligated into Invitrogen's pCR-Blunt (K270020) plasmid using T4 DNA Ligase (New England BioLabs, M0202S), at $4^{\circ} \mathrm{C}$ overnight. MIR196A enhancers and promoters were digested from pCR-Blunt and cloned into the luciferase reporter plasmid pGL3-Basic (Promega, E1751). Enhancers were cloned into the BamHI/Sall site whilst promoters were cloned into the multiple cloning site immediately upstream of the luciferase gene. See Supplementary Table 1 for primers.

MCF7 cells were transfected in antibiotic free media with $500 \mathrm{ng}$ of modified pGL3 reporter constructs, $20 \mathrm{ng}$ of pRL-TK (Renilla transfection control) and with $0.5 \mu \mathrm{L}$ of Lipofectamine 3000 (Life Technologies, L3000-008). $48 \mathrm{~h}$ post transfection luciferase readings were measured using a DTX-880 luminometer and Dual-Glo Stop and Glo luciferase reporter kit (Promega, E2920), following the manufacturer's recommended protocol.

RNA extraction and gene expression

Cell lysates were prepared using Life Technologies TRIzol $^{\circledR}$ reagent and RNA was chloroform extracted and isopropanol precipitated. RNA was DNasel treated with the DNA free kit from Ambion (Life Technologies, AM1906). RNA for miRNA analysis was reverse transcribed using the miScript RT II kit from Qiagen (218161), following instructions as per the manufacturer. Assays for all miRNAs were performed with Qiagen's miScript SYBR Green PCR Kit (218073). Primers specific to each mature or precursor miRNA were assayed coupled with a universal primer, see Supplementary Table 2 for assay IDs. Expression data for miRNAs was normalised to the snoRNA RNU6b. All qRT-PCRs were performed using the protocols advised by the manufacturers on a Corbet Rotorgene-6000.

Processed read counts for RNA-Seq on MCF7 cells following oestradiol treatment was sourced from K. Nephew (see author list). ${ }^{23}$ RNA-Seq from Adriamycin (ADM) and paclitaxel (PTX) resistant MCF7 derived cells was sourced from GSE68815, ${ }^{24}$ as processed and normalised read counts. Expression of HOX genes in human breast cells was sourced from Gascard et al. $^{25}$ as normalised read counts.

Genomic data analysis

Accession codes for publicly available data were as follows, MCF7 ChIP-Seq (GSE14664, ${ }^{26}$ ), GRO-Seq (GSE27463, ${ }^{27}$ ), ChIA-PET $\left(\right.$ GSE39495, $\left.{ }^{28,29}\right)$, Breast tumour ERa ChIP-Seq (GSE32222, ${ }^{30}$ ). MCF7 histone ChIP-Seq and breast cell $450 \mathrm{~K}$ array data was sourced from ENCODE $^{31}$ via http://genome.ucsc.edu/ENCODE/downloads. html. ChIP-Seq reads were adapter trimmed and data was mapped to the human genome (hg19) using Bowtie ${ }^{32}$ and peaks called by $\mathrm{MACS}^{33}$ and viewed in the Interactive Genome Viewer (IGV) ${ }^{34}$ available through the Broad Institute servers. DNA methylation $450 \mathrm{~K}$ array data for MCF7 and endocrine resistant sublines was previously published, see Stone et al. ${ }^{35}$ Normalised DNA methylation of breast tumours was sourced from The Cancer Genome Atlas (TCGA). ${ }^{36}$ Methylation $\beta$-values were correlated to the gene expression of MIR196A from the TCGA cohort, ${ }^{36}$ Pearson correlation coefficients are reported. For transcription factor (TF) binding to the MIR196A2 promoters, the genomic regions upstream of the MIR196A2 gene were visualised through the UCSC genome browser. $^{37}$ ENCODE $^{38}$ TF ChIP-Seq and JASPAR ${ }^{39}$ TF motifs were mapped across the putative promoter elements and snapshots from the browser were taken.

\section{Breast tumour expression analysis}

METABRIC expression and clinical information were sourced from EGAS00000083 through a Material Transfer Agreement with the consortium. ${ }^{40,41}$ Expression values were pre-processed by METABRIC and available as log2 array intensities. Clustering of Illumina Array and miR-Seq data was performed using the Multiple Experiment Viewer $\left(\mathrm{MeV}_{1}^{42}\right)$. Data was mean-centred and hierarchically clustered via Manhattan average-linkage based clustering of both rows and columns. Genes were correlated within clusters using the CORREL function of Microsoft Excel. The protein network was generated through the cBioPortal link (www. cbioportal.org, ${ }^{43}$ ) using the TCGA data. ${ }^{36}$ cBioPortal utilises protein data and visualisation tools through Cytoscape. ${ }^{44}$

\section{Survival analysis}

Tumour cohorts were based on immunohistochemistry of METBARIC ${ }^{40}$ patients as either ER + or ER- PGR- HER2 - as triple negative breast cancer (TNBC). Univariate and multivariate Cox proportional hazard regression analyses were performed using MedCalc for Windows, version 12.7 (MedCalc Software, Ostend, Belgium). Kaplan-Meier survival analysis and generation of survival curves was done in GraphPad Prism. Optimal cutoffs for low and high expression groups were determined using receiver operator characteristic (ROC) curves based on the expression of genes (MIR196A, HER2 and PGR) versus patient overall survival. The gene expression value that represents the maximum deviation from the 'random guess' line was used as a cut-off to discriminate low versus high expression. Lymph node status was designated as positive $(+, \geq 1$ node presenting with disease at time of surgery) or negative $(-)$. Tumour grade and stage clinical information were sourced from METABRIC. ${ }^{40}$ Tumour size was categorised as T1 = $20 \mathrm{~mm}, \mathrm{~T} 2=>20 \mathrm{~mm}$, and $<50 \mathrm{~mm}$ and $\mathrm{T} 3=\geq 50 \mathrm{~mm}$.

\section{C and ChIA-PET}

Chromosome conformation capture (3C) was adapted from Vakoc 2005, ${ }^{45}$ Hagege $2007^{46}$ and Tan-Wong 2008. ${ }^{47}$ Briefly, cells were grown to $60-80 \%$ confluencey and fixed with $1 \%$ paraformaldehyde. Libraries were generated for each cell line using HindIII with control libraries undigested and unligated, representing native gDNA without chromosome conformation. 
GAPDH primers (amplified fragment contains no cut sites for these enzymes) were used to determine the digestion and ligation efficiency of each library by comparing 3C-qPCR values to primers that amplify a fragment containing a Hindlll cut site. For each 3C-qPCR, primers were designed between 100-250 bp up or downstream of each Hindlll cut site with the primer across the putative enhancer used as bait in each 3C-qPCR. The bait primer was combined with each of the primers across the enhancer region for the $3 C-q P C R$ and $C t$ levels from each $3 C$ QPCR were normalised to the lowest $\mathrm{Ct}$ value (most abundant interaction) so that this interaction $=1$ relative interaction. Enhancer-promoter interactions are demonstrated as a peak across a region of multiple primers.

\section{RESULTS}

MIR196A expression correlates with HOXC genes in breast cancer Several HOXC protein coding and non-coding genes have known associations with breast cancer progression. We assessed expression patterns of HOXC genes and MIR196A (mature miRNA) in the METABRIC cohort of breast tumours (Supp Fig. 1). These data indicate that MIR196A expression highly correlates to HOXC genes, particularly HOXC10, which lies directly upstream of this miRNA.

Next, we investigated whether these associations are also observed in normal cells of the human breast. Here associations between mature MIR196A expression and HOXC genes are more limited, with correlations most strongly with $\mathrm{HOXC11}$ and $\mathrm{HOXC10}$, the genes upstream of the HOXC MIR196A2 gene (Supp Fig. 2A). Consistent with its role in degrading $\mathrm{HOX}$ transcripts, $\mathrm{HOXC8}$, $H O X B 8$ and HOXA7 (all validated targets) negatively correlate with MIR196A expression. MIR196A appears to be most highly expressed within the basal stem-cell (BSC) derived cells, whilst expression is lower in the more differentiated cell types (Supp Fig. 2B).

MIR196A expression is regulated by oestrogen

We and others have previously demonstrated regulation of $H O X C$ genes by oestrogen in breast cancer. ${ }^{48-52}$ Given that MIR196A expression strongly correlates with expression of HOXC protein coding genes in breast tumours (Supp Fig. 1), we sought to determine if oestrogen also regulates the HOXC embedded MIR196A2 precursor gene. Chromatin immunoprecipitation (ChIP-Seq) for RNA polymerase II demonstrates that polymerase binding in the region surrounding the HOXC10 gene and MIR196A gene is dependent on oestrogen in MCF7 cells (Fig. 1a). Globalrun-on sequencing (GRO-Seq) is able to measure nascent RNA, assessing changes in transcription with high sensitivity. Analysis of MCF7 GRO-Seq data clearly indicates a dramatic increase in RNA production in the genomic region surrounding MIR196A2, peaking at 40 mins following addition of oestradiol (E2) (Fig. 1b). This increase in RNA production from the HOXC locus was validated with qRT-PCR from MCF7 cells following addition of E2 (Fig. 1c). The regulation of $\mathrm{HOXC10}$ by oestrogen has been previously established, ${ }^{51}$ we find similar results which indicate an increase in expression by E2 (Supp Fig. 3). We next analysed data from MCF7 cells where low levels of E2 ( $1 \mathrm{nM})$ were used and find a similar pattern of a rapid increase in pre-MIR196A2 expression (Fig. 1d). Additionally, there was no change in the expression of the HOXB precursor, pre-MIR196A1. Taken together this suggests that MIR196A is transcriptionally regulated by oestrogen through its HOXC precursor, MIR196A2.

Transcriptional regulation of the MIR196A2 precursor gene To identify the structural elements associated with the transcriptional regulation of MIR196A2, histone methylation patterns in the MCF7 breast cancer cell line were assessed. This analysis uncovered putative promoter elements upstream of MIR196A2 including a shared promoter with HOXC10 (Fig. 1a). Given the strong association of MIR196A and HOXC10 expression in breast and their co-regulation by oestrogen, it seems likely they may share a common promoter element which we have cloned in three separate elements labelled putative promoter 1 (PP1), Overlap (between PP1 and PP2) and PP2.

Given that MIR196A2 expression is regulated by oestrogen we hypothesised that its transcription may be controlled by the oestrogen receptor (ER). Using publicly available datasets we established that oestrogen mediated upregulation of MIR196A2 expression is accompanied by binding of ERa and its pioneer factor FOXA1 to two putative promoter regions, PP1 and PP3, upstream of the MIR196A2 transcription start site (Fig. 1b).

The putative promoter elements were subsequently cloned into luciferase reporter vectors to assess transcriptional activity. PP1 and PP2 (modestly) increased luciferase gene transcription (Fig. 1e), with the most active promoter in MCF7 cells being PP1 (HOXC10 promoter).

Given that ERa often binds to distal enhancer elements to exert its function, we examined the hypothesis that MIR196A2 is controlled by long-range transcriptional regulation, mediated by ERa tethered gene looping. Using ChIA-PET (Chromatin Interact Analysis by Paired End Tags) genome-wide chromatin interactions that immunoprecipitate with either ERa or RNA Polymerase II (correlative with active promoters and enhancers), we identified two major sites of interaction with the MIR196A2/HOXC10 promoters (Fig. 2a). One of these is a previously identified HOTAIR enhancer (HOTAIR distal enhancer, $\mathrm{HDE}^{49}$ ) and the other a novel interacting partner (MIR196A2-Enhancer, mE). Chromosome conformation capture ( $3 C$ ) enzymatic digestion of the HOXC genomic locus results in two fragments covering the MIR196A2 region. 3C-qPCR analysis demonstrates that both enhancer elements physically interact with each of the MIR196A2/HOXC10 promoter regions (Fig. 2b). Cloning of these fragments downstream of the putative-promoter luciferase reporters clearly demonstrates significant augmentation of transcription for both the PP1 and PP2, with HDE appearing to be the most active in MCF7 cells (Fig. 2c).

Interestingly, a previous study ${ }^{10}$ identified a SNP (rs11614913) and an upstream CpG island that are both associated with a decrease in breast cancer risk. This SNP lies within the MIR196A2 gene and the CpG island (CpG_Hoffman) is immediately upstream, falling into the $3^{\prime}$ end of the PP3. Analysis of DNA methylation reveals that this $\mathrm{CpG}$ island is mostly methylated in non-malignant MCF10A and cancerous MCF7 cells, whilst unmethylated in human mammary epithelial cells (HMEC) (Fig. 1a).

MIR196A is differentially expressed in breast cancer Since MIR196A is regulated by ERa, we investigated its expression patterns in relation to commonly utilised molecular markers of breast tumours (Fig. 3a). This analysis identified four distinct clusters of MIR196A expression (Clusters 1-4). Interestingly clusters 1 and 3 show a strong correlation to expression of hormone receptors (HR) (AR, ERa, PGR, HER2) and HR cofactors (Fig. 3b). In contrast, clusters 2 and 4 have significant negative correlation to expression of ERa, PGR, FOXA1 and GATA3, whilst associating with EGFR and HER2. This expression is further defined by the PAM50 intrinsic subtypes where MIR196A is strongly expressed in the HER2 subtype, whist in the luminal A and B subtypes expression is very dynamic (Fig. 3c).

DNA methylation accumulates within promoters at $\mathrm{CpG}$ islands to suppress gene expression through inhibition of transcription factor binding. ${ }^{53}$ To predict further regulators of MIR196A expression in clusters 2 and 4 where negative correlation to ESR1 is seen, we investigated the DNA methylation of our putative promoter elements. The majority of upstream $\mathrm{CpGs}$ show a negative correlation to MIR196A expression, which the strongest correlation seen to sites within PP2 and A2-Gene (Fig. 3d). Utilising ENCODE TF ChIP-Seq data and motif sites from JASPAR, we identified 49 TFs binding within $100 \mathrm{bp}$ of these methylation sites 


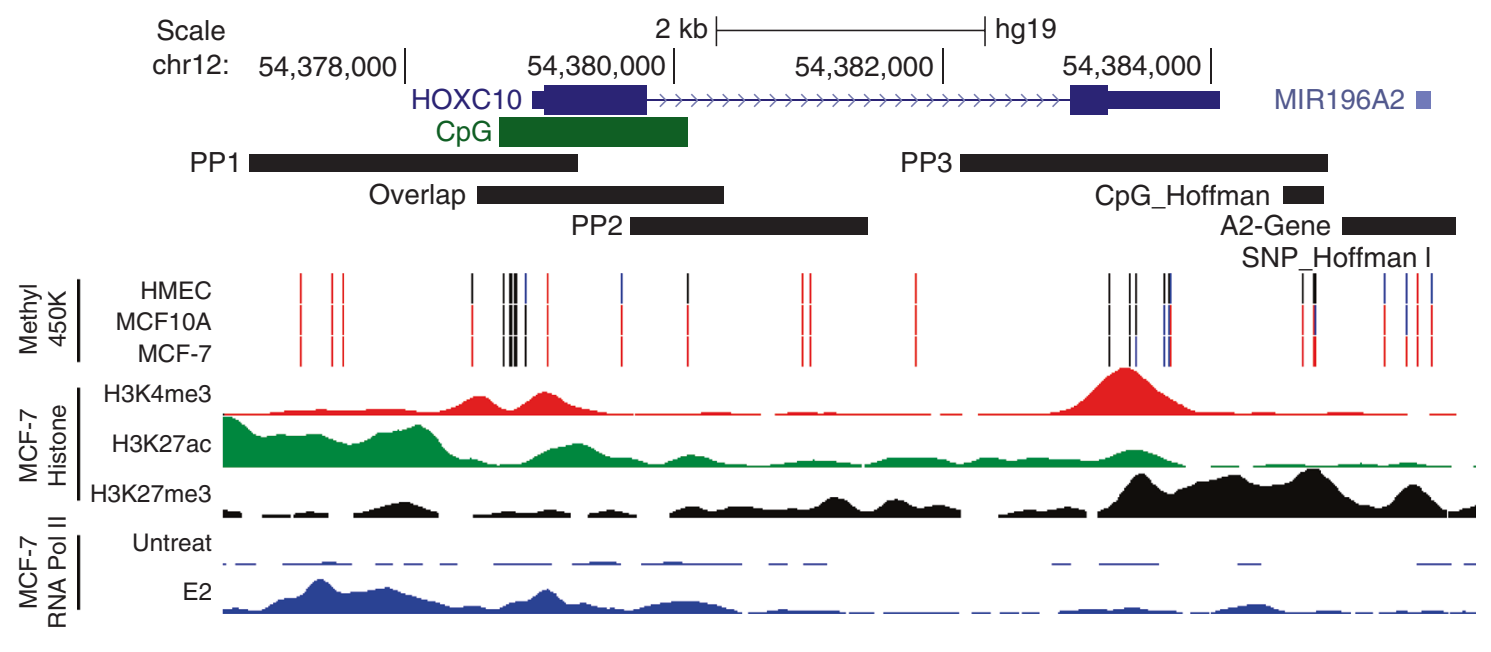

b

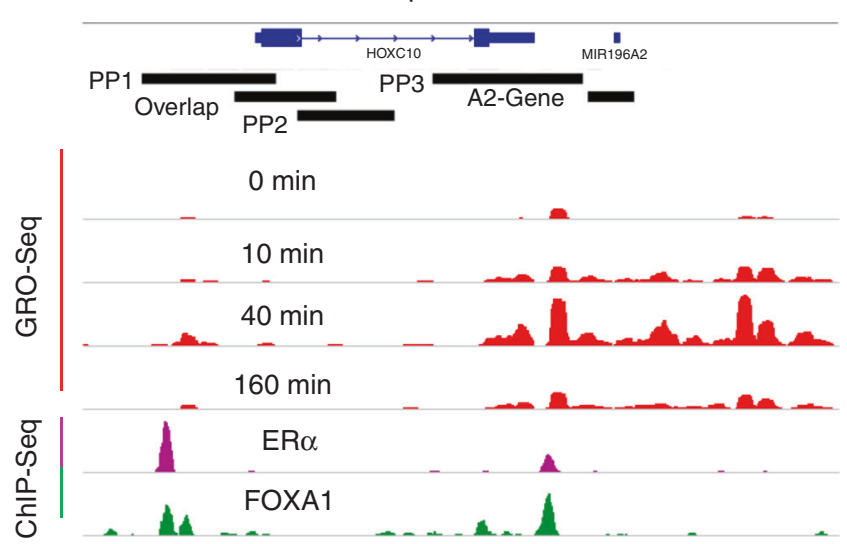

C

miR-196a expression

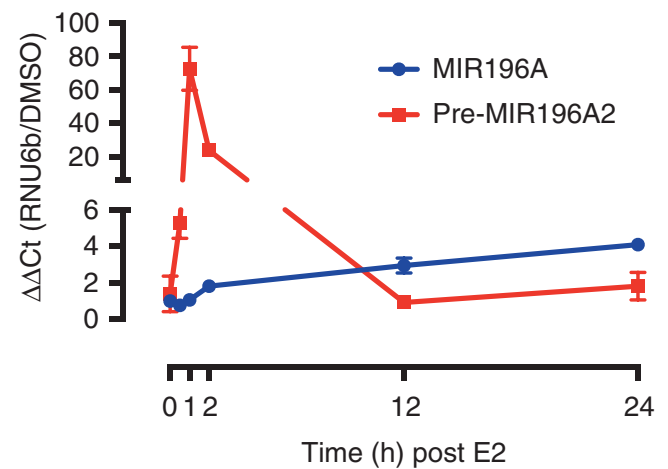

MCF7 MIR196A2

putative promoters
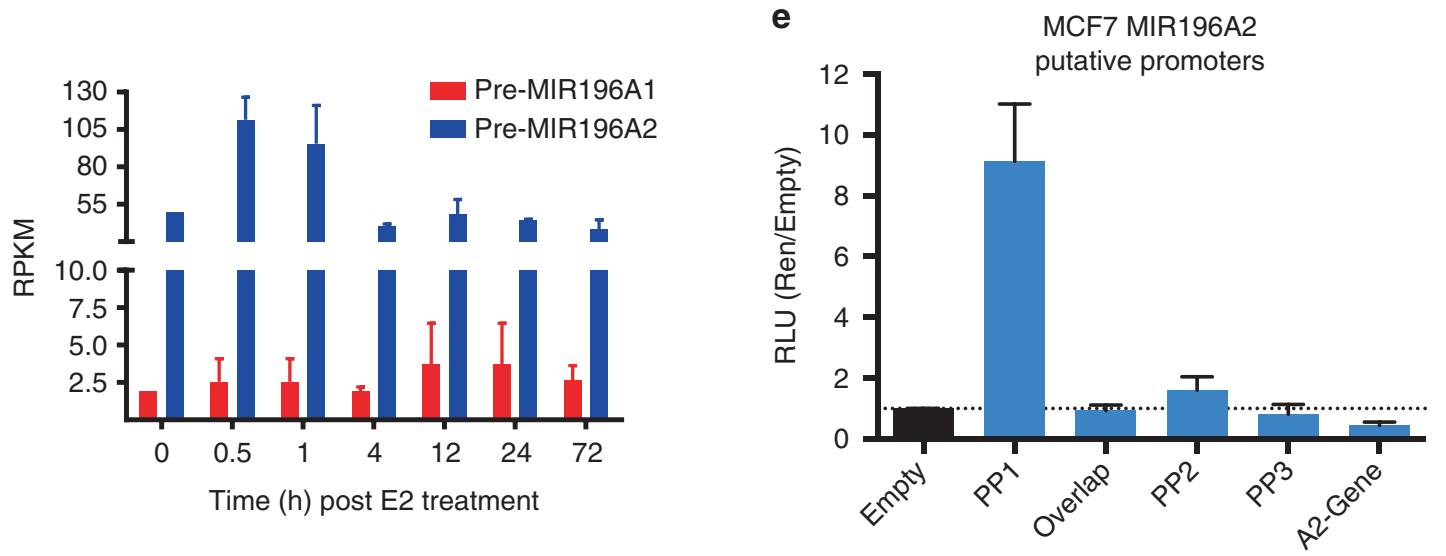

Fig. 1 E2 influences MIR196A2 expression in breast cancer. a Identification of putative promoter regions for the MIR196A2 gene using histone marks and ChIP-Seq indicated in figure. Refseq genes are indicated in blue at the top with coordinates based on hg19 chromosome 12. Putative promoter regions (PP1,2,3) and the previously implicated SNP (rs116149130) and CpG from Hoffman et al. ${ }^{10}$ are indicated by black rectangles. MCF7 DNA methylation $450 \mathrm{~K}$ array data indicate unmethylated (black), partial methylation (blue) and methylated (red). b GRO-Seq measurements of RNA Polymerase engagement and elongation points from the putative promoters, after E2 stimulation in MCF7 cells. Lower part, ChIP-Seq for binding of ER $\alpha$ and FOXA1 to the putative promoters. c qRT-PCR the MIR196A2 response to E2 in MCF7 cells. Qiagen precursor primers were used to detect the precursor miRNA at the specified time points and CT values were normalised to a DMSO vehicle control and the qRT-PCR control of RNU6b. d MiRNA-Seq RPKM for the precursor miRNAs following E2 addition to MCF7 cells. e Luciferase reporter assay measuring the influence of MIR196A2 putative promoter on the luciferase gene transcription. Measurements are RLU normalised to the renilla plasmid (pRL-TK) acting as a transfection control and to the $\mathrm{pGL3/Empty} \mathrm{plasmid.} \mathrm{Experimental} \mathrm{measures} \mathrm{are} \mathrm{done} \mathrm{in}$ triplicate with the experiment repeated, data not shown. E2 oestradiol, H3K4me3 Histone 3 Lysine 4 tri-methylation, H3K27ac Histone 3 Lysine 27 acetylation, H3K27me3 Histone 3 lysine 27 tri-methylation, HMEC Human Mammary Epithelial Cell, GRO-Seq Global run-on sequencing, RPKM reads per kilobase per million, RLU relative light units 

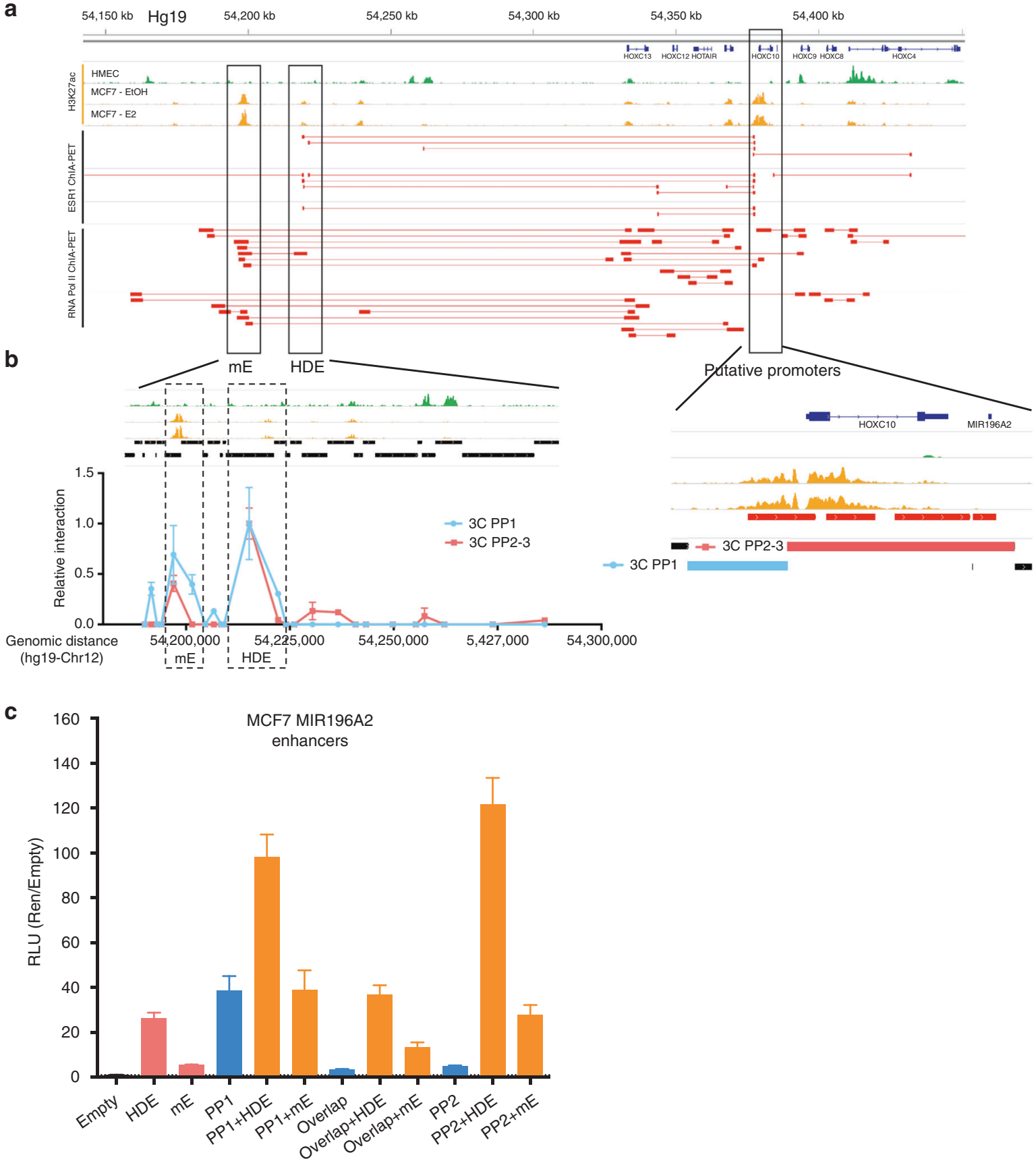

Fig. 2 Distal putative enhancer elements of the MIR196A2 putative promoters. a Histone modification and ChIA-PET of ESR1 and RNA Pol II in MCF7 cells across the HOXC locus and corresponding gene desert. The histone modification H3K27ac is a measure of regulatory element activity and was assessed in HMEC and MCF7 cells plus or minus E2. ChIA-PET interactions are represented by red lines, solid rectangles indicate the sequenced tag and the two points that were physically interacting and tethered to either ESR1 or RNA Pol II. b Zoom-in of mE and HDE (left) and the putative promoter elements (right). Black rectangles indicate the genome fragment sizes post digestion with Hindlll. Graph is 3C-qPCR for either the 3 C PP1 or 3 C PP2-3 fragments with the $Y$-axis the relative interaction and the $X$-axis the genomic location. All genomic coordinates were based on chromosome 12 in the hg19. c Luciferase reporter assay showing the augmentation of HOXC promoters with either mE or HDE, graphed as RLU normalised to the co-transfected control renilla plasmid and to the vector backbone pGL3-Basic. ChIA-PET Chromatin interaction analysis by paired-end tags, HDE HOTAIR distal enhancer, mE MIR196A2 enhancers, PP1/2/3 putative promoter 1

(Supp Figs. 4 and 5, Supp Table 3). Of the 49 TFs, 18 factors exhibit significant positive correlation in clusters 2 and 4, while 9 are significantly negatively corelated to MIR196 A expression (Supp Fig. 6A, Supp Table 3). Interestingly, a cluster of factors (CEBPA, CEBPB, EBF1, EGR1, EGR2, EZH2, JUN, KLF4, KLF5, PPARG, RXRA) presents as highly interconnected through protein-protein interactions and transcriptional regulation and appears largely

b 
a

Mean centred expression

$-3$
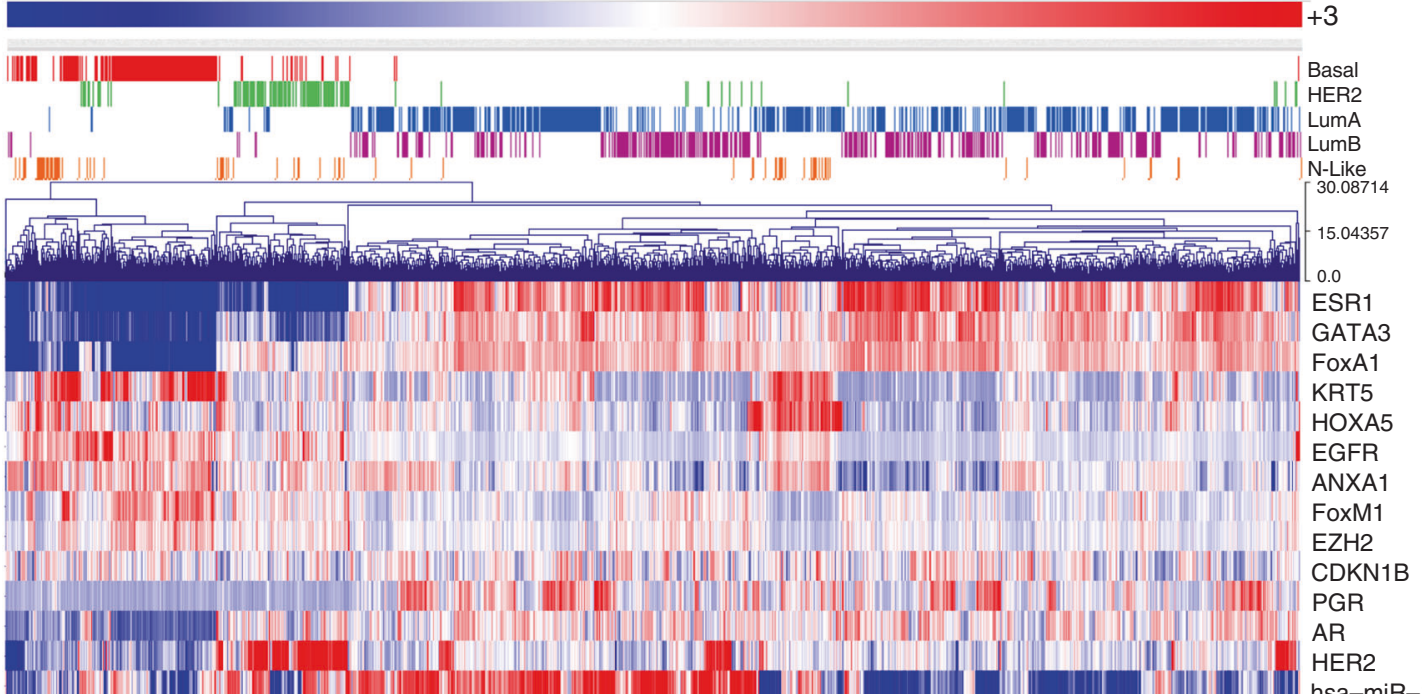

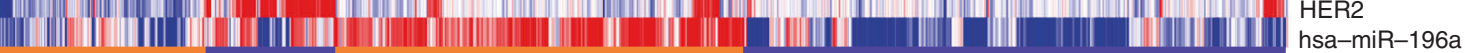

12

b

\begin{tabular}{|l|c|c|c|c|}
\multicolumn{4}{c}{ Correlation to MIR196A } \\
\cline { 2 - 5 } \multicolumn{1}{c|}{ Cluster 1 \& 3 } & \multicolumn{2}{c|}{ Clusters 2 \& 4 } \\
\hline Gene & Pearson & Pvalue & Pearson & Pvalue \\
\hline CDKN1B & -0.010 & $8.1 \mathrm{E}-01$ & -0.113 & $3.3 \mathrm{E}-03$ \\
\hline EGFR & -0.494 & $1.1 \mathrm{E}-39$ & 0.283 & $8.0 \mathrm{E}-14$ \\
\hline EZH2 & -0.189 & $2.1 \mathrm{E}-06$ & 0.043 & $2.6 \mathrm{E}-01$ \\
\hline KRT5 & -0.424 & $1.3 \mathrm{E}-28$ & 0.207 & $6.5 \mathrm{E}-08$ \\
\hline HOXA5 & -0.201 & $4.0 \mathrm{E}-07$ & 0.147 & $1.3 \mathrm{E}-04$ \\
\hline ANXA1 & -0.402 & $1.3 \mathrm{E}-25$ & 0.260 & $7.6 \mathrm{E}-12$ \\
\hline GATA3 & 0.653 & $3.8 \mathrm{E}-77$ & -0.324 & $6.1 \mathrm{E}-18$ \\
\hline ESR1 & 0.647 & $2.0 \mathrm{E}-75$ & -0.413 & $4.8 \mathrm{E}-29$ \\
\hline AR & 0.548 & $3.7 \mathrm{E}-50$ & 0.031 & $4.3 \mathrm{E}-01$ \\
\hline Foxa1 & 0.711 & $2.0 \mathrm{E}-97$ & -0.167 & $1.4 \mathrm{E}-05$ \\
\hline FoxM1 & -0.316 & $6.8 \mathrm{E}-16$ & -0.020 & $6.1 \mathrm{E}-01$ \\
\hline PGR & 0.455 & $3.4 \mathrm{E}-33$ & -0.228 & $2.4 \mathrm{E}-09$ \\
\hline HER2 & 0.377 & $1.9 \mathrm{E}-22$ & 0.291 & $1.4 \mathrm{E}-14$ \\
\hline
\end{tabular}

C

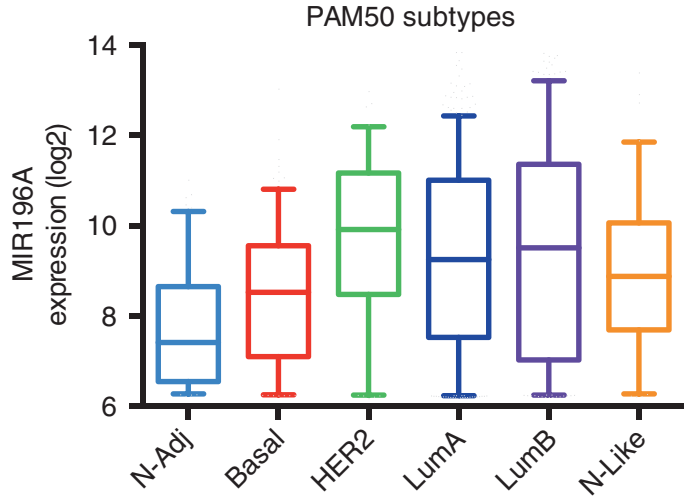

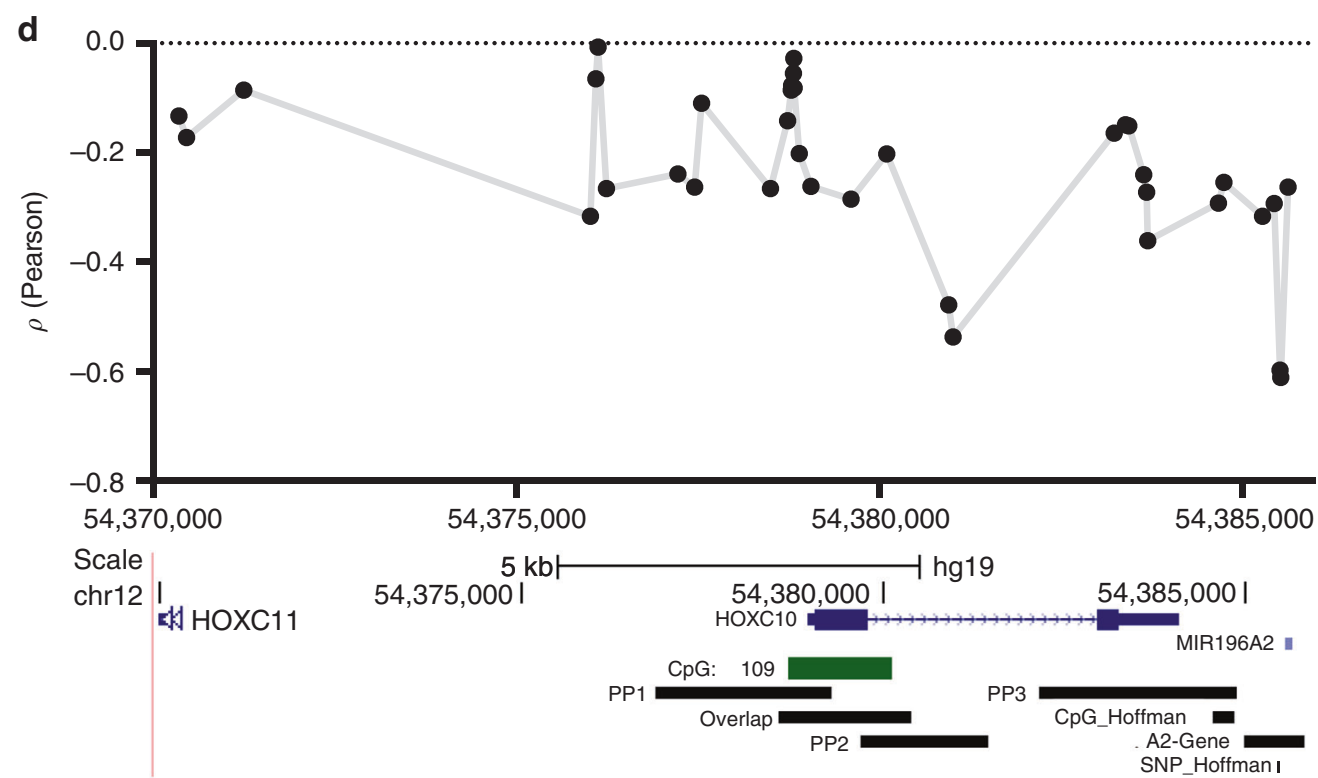

tumours. ${ }^{40,41}$ Expression analysis of this miRNA indicate that it is significantly over-expressed in breast tumours compared to normal adjacent tissue and over-expression is associated with an increase in tumour stage (Fig. 4a, b). Interestingly, high expression of MIR196A is associated with a poor survival in oestrogen receptor positive $(\mathrm{ER}+)$ breast cancer, whilst high expression associates 
Fig. 3 MIR196A is differentially expressed in breast cancer. a Mean-centred log2-expression of MIR196A and commonly utilised breast cancer molecular markers. Expression values were hierarchically clustered and the PAM50 tumour subtypes are indicated above the plot. Expression values are indicated by colour scale bar. b Pearson correlation coefficients, and corresponding P-values, for each gene against the expression of MIR196A either in the orange or purple clusters. c Intensity values for the expression of MIR196A across the five molecular subtypes, PAM50. d Correlation of HOXC methylation to expression of MIR196A for CpG dinucleotides upstream of its gene. Data was sourced from the METABRIC cohort $^{40,41}$ for $\mathbf{a}, \mathbf{b}$ and $\mathbf{c}$. Methylation and expression data for $\mathbf{d}$ was sourced from the TCGA cohort of breast tumours. ${ }^{36}$ Basal $(n=179)$, HER2 (HER2-enriched, $n=112$ ), LumA (Luminal A, $n=568$ ), LumB (Luminal B, $n=354$ ), N-Adj (Normal-adjacent, $n=116), \mathrm{N}$-Like (Normal-like, $n=82$ )

with a better outcome in triple-negative breast cancer (TNBC) over the first 5 to 10 years following initial diagnosis (Fig. 4c, d).

Using MIR196A expression, overall survival of ER + tumours responding to both hormone therapy $(\mathrm{HT})$ and chemotherapy $(\mathrm{CT})$ was stratified (Fig. 4e). Women with low MIR196A expression had exhibited a high rate of survival $(>95 \%$ at 10 years, $\mathrm{HR}=8.003, P$ value $=0.0125)$, whilst most women within the high expression group died within 17 years (61\% at 10 years).

Given that MIR196A is regulated in part by oestrogen, and the disparity in prognostication of ER+ and TNBC, we investigated the effects of menopause on the stratification of survival for ER+ women. The effects of menopause on the human breast are largely unknown, however serum levels of oestrogen and progesterone dramatically reduce post menopause. In pre-menopausal women, high expression of MIR196A is associated with improved overall survival in $E R+$ disease $(H R=$ $0.463, P$-value $=0.0288$ ) (Table 1 , Supp Fig. 7A). Multivariate analysis demonstrates that MIR196A is one of the few significant biomarkers for ER+ tumours arising before menopause. In postmenopausal women, all tested biomarkers were significant in $\mathrm{ER}+$ disease, including MIR196A, however high expression is now associated with decreased overall survival $(H R=1.847$, $P$-value $=0.0005)$ (Table 1, Supp Fig. 7B). A similar trend was also observed in TNBC, where in pre-menopausal women, MIR196A high expression correlates with a better outcome (Supp Fig. 7C), stratification in post-menopausal women however, found no significant trend (Supp Fig. 7D).

Therapeutic resistance leads to increases in MIR196A expression TNBC is resistant to hormone-based therapies and HR+ disease often becomes resistant to anti-oestrogen treatment. Using established models of $\mathrm{HR}+$ disease resistance we found that MIR196A expression is significantly increased in tamoxifen resistant MCF7 cells (TAMR) whilst it is almost depleted in acquired fulvestrant resistance (FASR) (Fig. 5a). These expression patterns match changes in DNA methylation to the HOXC10/ MIR196A2 promoters in these same cells (Fig. 5b). For HR+ resistant tumours the only remaining therapeutic options are radiotherapy and chemotherapy. Using RNA-Seq data for cell line models of resistance to adromycin (ADM) and paclitaxel (PTX), two commonly used chemotherapeutics, MIR196A expression again increases in resistant cell lines compared to the treatment sensitive cell line (Fig. 5c). These data suggest an intrinsic requirement for elevated MIR196A expression in $\mathrm{HR}+$ tumour resistance.

Several HOX genes are validated targets of MIR196A (HOXA7, HOXB8, HOXC8 and HOXCD8) HOXA7 shows the greatest negative correlation to MIR196A in human breast cells (Supp Fig. 2A). Expression of HOXA7 strongly mirrors that of MIR196A in the panel of endocrine resistant MCF7 sublines (Fig. 5d). Additionally, expression of HOXA7 decreases in the ADM and PTX resistant lines (Fig. 5e), in contrast to the increase in MIR196A expression. In these models of therapeutic resistance, MIR196A may be reducing expression of HOXA7.

Utilising ERa ChIP-Seq performed in human patients with $\mathrm{HR}+$ disease, ${ }^{30}$ binding sites for ERa were identified in the genomic region of MIR196A. This tumour cohort contains three groups of tumours, (1) tumours from women who respond to HR therapy, (2) those who do not and (3) metastases from resistant tumours. An increase in ERa occupancy is seen at both enhancer and promoter regions of MIR196A in non-responders and metastases (Fig. 5f). The increased genome-wide ERa binding in the more resistant tumours was shown by the authors to associate with changes to expression patterns crucial for the resistant tumour to survive therapy and become resistant.

\section{DISCUSSION}

The expression of MIR196A in breast cancer is both dynamic and complex. In this paper, we have elucidated important elements, factors and mechanisms controlling the transcriptional regulation of MIR196A and shown that changes in regulation are associated with breast cancer progression and therapeutic resistance.

Several studies have demonstrated regulation of HOXC genes by oestrogen. ${ }^{4951,54}$ The majority of HOXC genes are lowly expressed in breast luminal epithelial cells (BLEC), where ERa is most highly expressed and cells are responsive to oestrogen. The regulation of HOXC genes by ERa may be specific to cancer cells through an acquired mechanism of regulation. Several studies have shown that enhancers that are normally repressed can become activated in cancer ${ }^{55,56}$ and given the extensive chromatin looping between the HOXC locus and its adjacent gene desert, this seems the likely mechanism for cancer expression.

We have previously demonstrated that long-range regulation of HOXC genes occurs in breast cancer and is influenced by ERa and its associated cofactors. ${ }^{49}$ HOX gene expression is tightly controlled in a spatiotemporal manner to ensure proper axial formation along the anterior-posterior axis during embryonic development. ${ }^{57}$ Within the cell types of the human breast, $\mathrm{HOX}$ gene expression appears dynamic and the association between MIR196A and HOXC genes is not significant. The strong correlation in expression of all HOXC genes in breast tumours with MIR196A is in stark contrast to expression in normal tissues. Several instances have been described regarding the influence of multiple distal enhancers on gene expression, such as the well characterised locus-control-region (LCR) of the Beta-globin genes or the c-Myc enhancers active across multiple cancer types. ${ }^{45,58-60}$ Given the extensive interactions between this locus and its adjacent gene desert, we hypothesise that a consorted effort of multiple enhancers is responsible for the overexpression of these genes in cancer possibly driven by extensive binding and activity of ERa. To explore this hypothesis a high resolution chromatin interaction analysis of this region in breast cancer cells would be required, such as $5 C^{61}$ or NG Capture-C, ${ }^{62}$ coupled with ERa ChIP-Seq and ChIA-PET. ${ }^{29}$ In addition, we see an increase in MIR196A2 expression in response to low-dose E2, suggesting a direct influence by ERa that is further increased at higher dosages of $10 \mathrm{nM}$ in our qRTPCR assays. It would be interesting to explore how low dosages of E2 influence distal enhaner elements and if more oestrogen is required for enhancer-promoter activity.

Whilst this manuscript was in preparation new data has come to light which corroborates our conclusions. Jiang et al. ${ }^{63}$ demonstrate that the mature MIR196A transcript positively responds to 
a

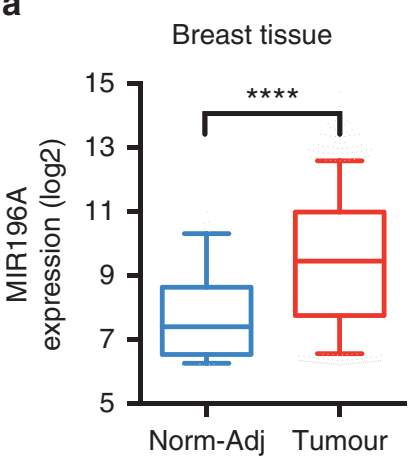

$T$ test, $P \leq 0.0001$

C

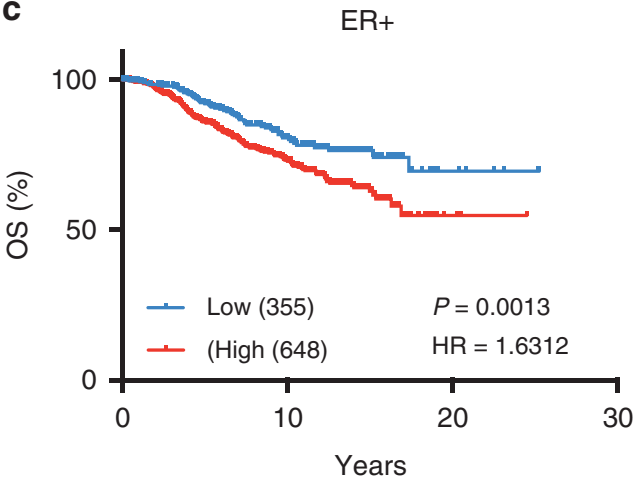

e

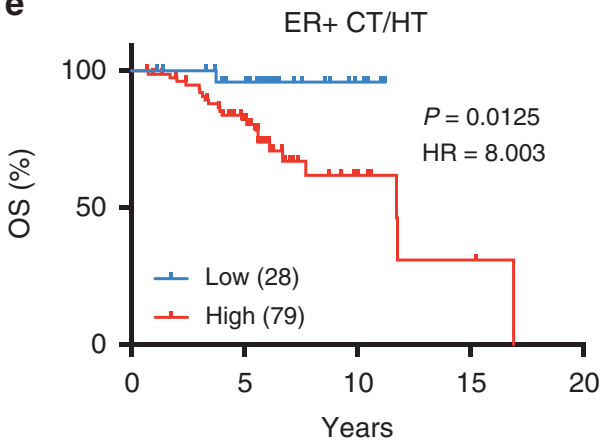

b

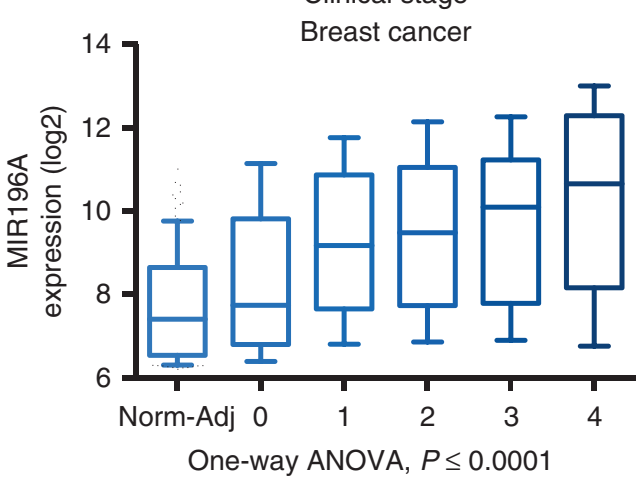

d

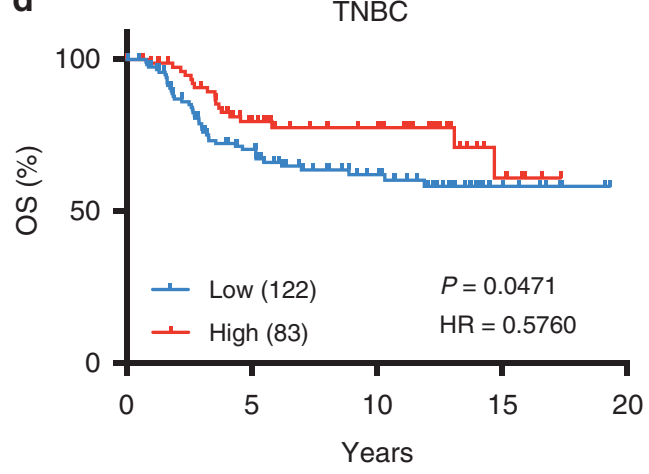

\section{0}

(1) 


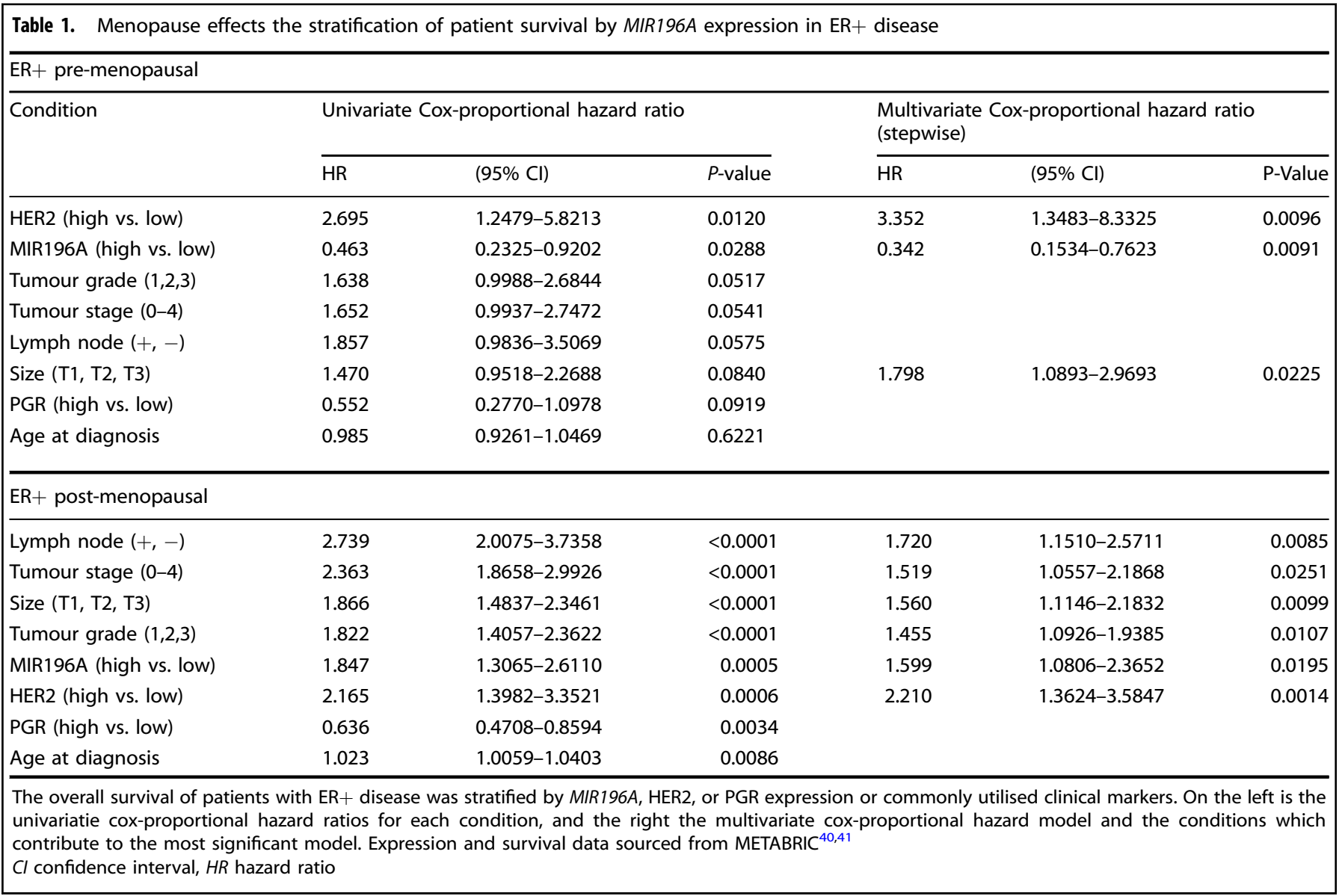

data suggest that binding of ERa accompanied by cofactors may be needed to maintain low methylation levels and active transcription in breast cancer. ${ }^{68-72}$

Using hierarchical clustering of breast tumour RNA-Seq data, we observed two distinct expression patterns associated with MIR196A expression. Interestingly, DNA methylation at several sites within the HOXC locus negatively correlates with the expression of this miRNA, supporting the notion of DNA methylation as a repressive epigenetic modification in this context. ${ }^{67}$ We demonstrated that several transcription factors that bind to these differentially methylated regions strongly associate with MIR196A expression in breast cancer, even in those tumours with show a negative correlation to ERa expression. These transcription factors appear to influence the expression of one another and in some cases form proteinprotein complexes. Further investigations should aim to fully elucidate the role of this network and its influence on MIR196A expression.

High expression of MIR196A is a biomarker of poor prognosis in $\mathrm{ER}+$ tumours, especially in those patients resistant to therapy. Expression of MIR196A increases in response to tamoxifen and chemotherapeutic agents in oestrogen responsive MCF7 cells. This increase in expression is associated with loss of DNA methylation within the promoter regions of the miRNA. In poor responders with ER+ tumours, HOXC enhancer elements appear to more readily bind the ER. These data raise the possibility that the pathway to resistance to therapy in ER+ tumours involves the derepression and over-activation of promoter and enhancer elements. This is commonly seen throughout cancer, ${ }^{56,73,74}$ with suggestions that enhancer disruption can revert cells to a nonterminally-differentiated state a common hallmark of tumourigenesis. HOX genes are essential in embryonic development, these genes would be a valuable asset for any tumour cell to use to sustain a stem-cell like state. ${ }^{75,76}$

Breast cancer incidence and relative subtype changes after menopause. ${ }^{77,78}$ In women younger than 45, luminal breast tumours account for $33-44 \%$. $^{79,80}$ This increases to $70-72 \%$ in women older than 65 . In contrast, basal-like tumours are more common in younger women, suggesting a switch or evolution in the factors driving cancer following menopause, most likely related to the decline in oestrogen production. It is then interesting to note that higher expression of MIR196A associates with good outcome in pre-menopausal women with ER+ tumours, and a poor outcome of ER+ tumours following menopause. Given the strong involvement of HOX genes in development, we hypothesise that there is a change in the regulation and expression of these genes through and following menopause, which in turn impacts their contribution to the development of certain breast cancer subtypes.

MIR196A is a dynamically expressed miRNA in both normal mammary cells and breast tumours. This miRNA is a possible biomarker for the progression of breast tumour to becoming resistant to therapy. Future studies should aim to uncover the purpose of increase MIR196A expression and if it is required for development of resistance alone or in combination with other HOXC genes. 


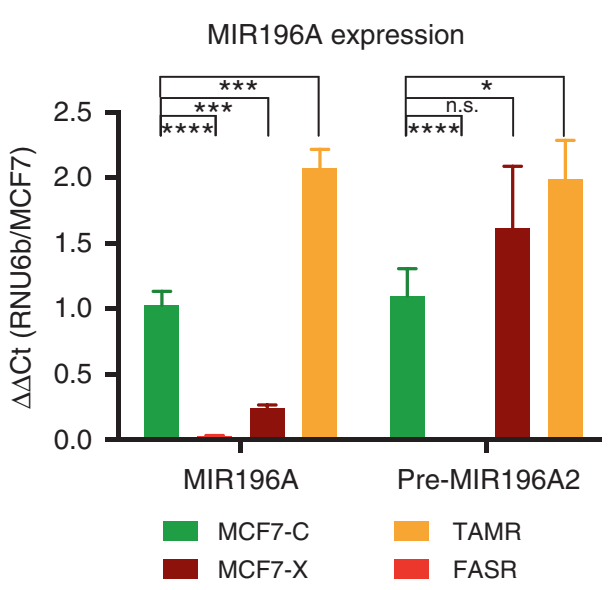

C

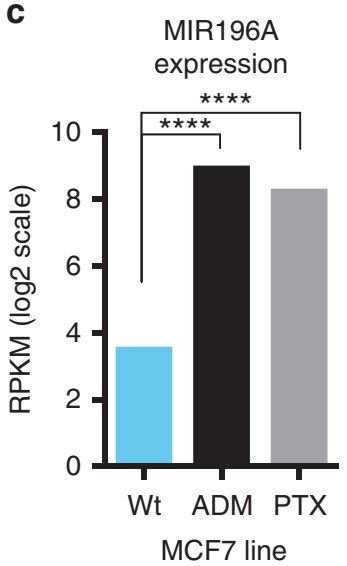

b

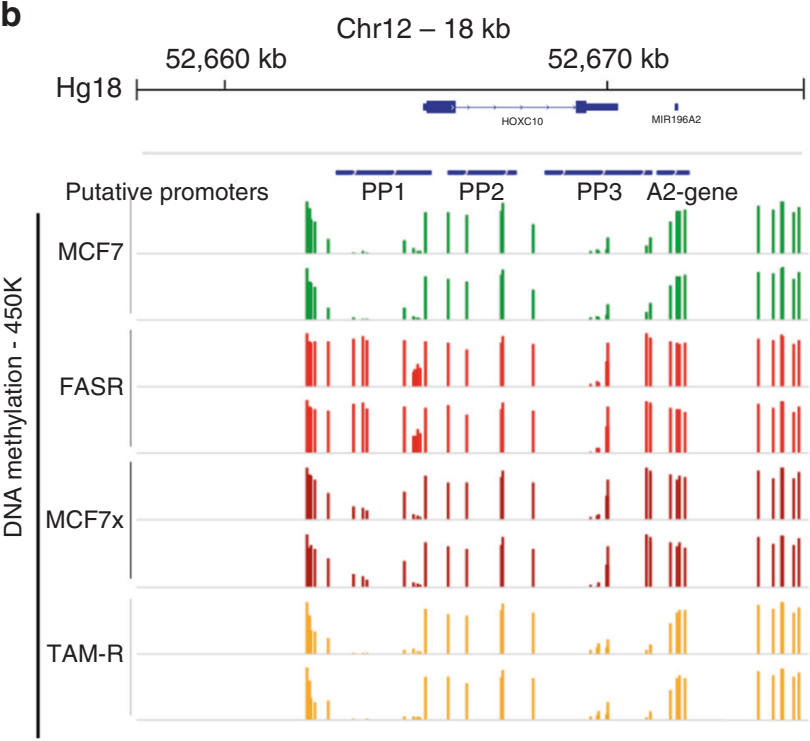

d

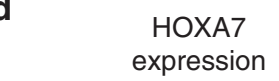

e

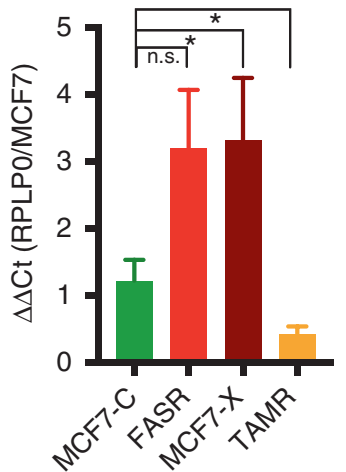

HOXA7 expression $\mathbf{f}$

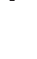

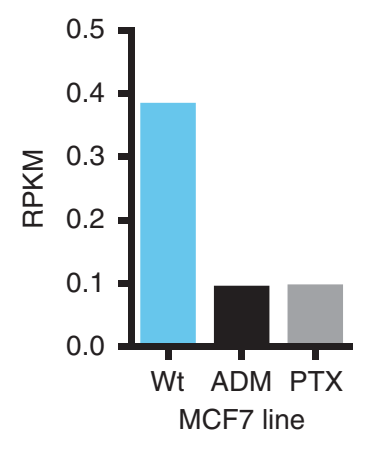

Tumour ER $\alpha$ ChIP

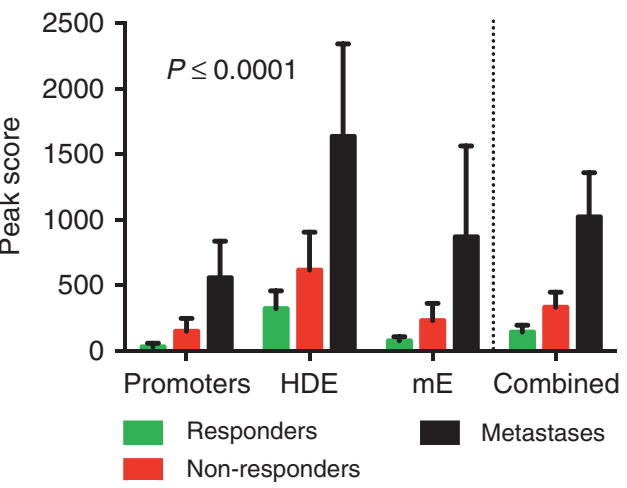

Fig. 5 Therapeutic resistance leads to an increase in MIR196A expression. a, d qRT-PCR of the relative expression for the mature MIR196A and precursor MIR196A2 transcripts and HOXA7 in MCF7 derived cell line models of endocrine therapy resistance. miRNA expression values are normalised to the expression of RNU6b and the MCF7-C cell line, HOXA7 expression was normalised to RPLPO and MCF7-C expression. Error bars are the standard deviation of two technical replicates and four biological replicates. b Corresponding DNA methylation for MCF7 derived cell lines, as measured by $450 \mathrm{~K}$ methylation array, for the MIR196A2 genomic region. c, e Log2 RPKM expression of MIR196A and HOXA7 in MCF7 wild-type and ADM and PTX derived resistance cell lines. $f$ Peak scores for the binding of ER $\alpha$ to MIR196A2 regulatory elements in ER+ breast tumours. Peak scores were generated using MACS, normalised to the Input control for the ChIP-Seq library. Peak scores are the average for 9 responders, 9 non-responders and 3 metastases. Data is sourced from Ross-Innes et al. ${ }^{30}$ ADM Adriamycin/doxorubicin, FASR Fulvestrant resistant, MACS model-based analysis for ChIP-Seq, MCF7-C Control, MCF7-X Oestrogen deprived, PTX paclitaxel, RPKM reads-per-kilobase-per-million, TAMR Tamoxifen resistant

\section{ACKNOWLEDGEMENTS}

This study makes use of data generated by the Molecular Taxonomy of Breast Cancer International Consortium (METABRIC). Funding for the project was provided by Cancer Research UK and the British Columbia Cancer Agency Branch. ${ }^{40,41}$ M.J.G.M. and M.A.B. were funded by the National Breast Cancer Foundation (2012002037). M. A.B., L.J.B. and S.C., received funding from the National Health and Medical Research Council (AP1058421 and AP1106907). Work by M.J.G.M., M.A.B., U.G., C.D.L.M., K.N. and L.J.B., was funded by the University of Queensland. J.M.W.G. was funded by the Tenovus Cancer Care Charity, a Breast Cancer Now Fellowship and Cardiff University. Funding for K.N. was provided by the National Institute of Heath (CA1130001).

\section{AUTHOR CONTRIBUTIONS}

M.J.G.M. and M.A.B. designed the study and wrote the manuscript. U.G., C.D.L.M., A.S., K.N., L.J.B., J.M.W.G., K.N. and S.C. contributed to experimental design, data and manuscript editing. All authors have agreed with the final version of the manuscript and provide their consent for publication.

\section{ADDITIONAL INFORMATION}

Supplementary information is available for this paper at https://doi.org/10.1038/ s41416-019-0395-8.

Competing interests: The authors declare no competing interests.

Data availability: Requests for data and reagents can be made by contacting the corresponding or senior authors.

Ethics approval and consent to participate: This study uses data previously obtained by the METABRIC and TCGA consortia and as such is not subjected to ethics approval or patient consent.

Publisher's note: Springer Nature remains neutral with regard to jurisdictional claims in published maps and institutional affiliations. 
MicroRNA-196a is regulated by ER and is a prognostic biomarker in ER..+ MJG Milevskiy et al.

\section{REFERENCES}

1. Wilczynska, A. \& Bushell, M. The complexity of miRNA-mediated repression. Cell Death Differ. 22, 22-33 (2015).

2. Iorio, M. V. \& Croce, C. M. MicroRNA dysregulation in cancer: diagnostics, monitoring and therapeutics. A comprehensive review. EMBO Mol. Med. 4, 143-159 (2012).

3. Simonson, B. \& Das, S. MicroRNA therapeutics: the next magic bullet? Mini Rev. Med. Chem. 15, 467-474 (2015).

4. Yekta, S., Tabin, C. J. \& Bartel, D. P. MicroRNAs in the Hox network: an apparent link to posterior prevalence. Nat. Rev. Genet. 9, 789-796 (2008).

5. Hornstein, E. et al. The microRNA miR-196 acts upstream of Hoxb8 and Shh in limb development. Nature 438, 671-674 (2005).

6. McGlinn, E. et al. In ovo application of antagomiRs indicates a role for miR-196 in patterning the chick axial skeleton through Hox gene regulation. Proc. Natl Acad. Sci. USA 106, 18610-18615 (2009).

7. Mansfield, J. H. et al. MicroRNA-responsive 'sensor' transgenes uncover Hox-like and other developmentally regulated patterns of vertebrate microRNA expression. Nat. Genet. 36, 1079-1083 (2004).

8. Yekta, S., Shih, I. H. \& Bartel, D. P. MicroRNA-directed cleavage of HOXB8 mRNA. Science 304, 594-596 (2004).

9. Hui, A. B. et al. Robust global micro-RNA profiling with formalin-fixed paraffinembedded breast cancer tissues. Lab. Investig. 89, 597-606 (2009).

10. Hoffman, A. E. et al. microRNA miR-196a-2 and breast cancer: a genetic and epigenetic association study and functional analysis. Cancer Res. 69, 5970-5977 (2009).

11. Luthra, R. et al. MicroRNA-196a targets annexin A1: a microRNA-mediated mechanism of annexin A1 downregulation in cancers. Oncogene 27, 6667-6678 (2008).

12. Hanahan, D. \& Weinberg, R. A. Hallmarks of cancer: the next generation. Cell 144 646-674 (2011).

13. Liu, X. H. et al. MicroRNA-196a promotes non-small cell lung cancer cell proliferation and invasion through targeting HOXA5. BMC Cancer 12, 348 (2012).

14. Sun, M. et al. MiR-196a is upregulated in gastric cancer and promotes cell proliferation by downregulatingp27(kip1). Mol. Cancer Ther. 11, 842-852 (2012).

15. Hou, T. et al. MicroRNA-196a promotes cervical cancer proliferation through the regulation of FOXO1 andp27(Kip1.). Br. J. Cancer 110, 1260-1268 (2014).

16. Wang, G. et al. RNA polymerase II binding patterns reveal genomic regions involved in microRNA gene regulation. PLOS ONE 5:e13798 (2010).

17. Corcoran, D. L. et al. Features of mammalian microRNA promoters emerge from polymerase II chromatin immunoprecipitation data. PLOS ONE 4, e5279 (2009).

18. Attema, J. L. et al. Identification of an enhancer that increases miR200b 200a 429 gene expression in breast cancer cells. PLoS ONE 8, e75517 (2013).

19. Punnamoottil, B., Rinkwitz, S., Giacomotto, J., Svahn, A. J. \& Becker, T. S. Motor neuron-expressed microRNAs 218 and their enhancers are nested within introns of Slit2/3 genes. Genesis 53, 321-328 (2015).

20. McClelland, R. A. et al. Enhanced epidermal growth factor receptor signaling in MCF7 breast cancer cells after long-term culture in the presence of the pure antiestrogen ICI 182,780 (Faslodex). Endocrinology 142, 2776-2788 (2001).

21. Knowlden, J. M. et al. Elevated levels of epidermal growth factor receptor/c-erbB2 heterodimers mediate an autocrine growth regulatory pathway in tamoxifenresistant MCF-7 cells. Endocrinology 144, 1032-1044 (2003).

22. Staka, C. M., Nicholson, R. I. \& Gee, J. M. Acquired resistance to oestrogen deprivation: role for growth factor signalling kinases/oestrogen receptor crosstalk revealed in new MCF-7X model. Endocr. Relat. Cancer 12(Suppl 1), S85-S97 (2005).

23. Miller, D. F. et al. A new method for stranded whole transcriptome RNA-seq Methods 63, 126-134 (2013).

24. He, D. X. et al. Genome-wide profiles of methylation, microRNAs, and gene expression in chemoresistant breast cancer. Sci. Rep. 6, 24706 (2016).

25. Gascard, P. et al. Epigenetic and transcriptional determinants of the human breast. Nat. Commun. 6, 6351 (2015).

26. Welboren, W. J. et al. ChIP-Seq of ERalpha and RNA polymerase II defines genes differentially responding to ligands. EMBO J. 28, 1418-1428 (2009).

27. Hah, N. et al. A rapid, extensive, and transient transcriptional response to estrogen signaling in breast cancer cells. Cell 145, 622-634 (2011).

28. Li, G. et al. Extensive promoter-centered chromatin interactions provide a topological basis for transcription regulation. Cell 148, 84-98 (2012).

29. Fullwood, M. J. et al. An oestrogen-receptor-alpha-bound human chromatin interactome. Nature 462, 58-64 (2009)

30. Ross-Innes, C. S. et al. Differential oestrogen receptor binding is associated with clinical outcome in breast cancer. Nature 481, 389-393 (2012).

31. Consortium, E. P. An integrated encyclopedia of DNA elements in the human genome. Nature 489, 57-74 (2012).
32. Langmead, B., Trapnell, C., Pop, M. \& Salzberg, S. L. Ultrafast and memory-efficient alignment of short DNA sequences to the human genome. Genome Biol. 10, R25 (2009).

33. Zhang, Y. et al. Model-based analysis of ChIP-Seq (MACS). Genome Biol. 9, R137 (2008)

34. Robinson, J. T. et al. Integrative genomics viewer. Nat. Biotechnol. 29, 24-26 (2011).

35. Stone, A. et al. DNA methylation of oestrogen-regulated enhancers defines endocrine sensitivity in breast cancer. Nat. Commun. 6, 7758 (2015).

36. Cancer Genome Atlas N. Comprehensive molecular portraits of human breast tumours. Nature 490, 61-70 (2012).

37. Rosenbloom, K. R. et al. The UCSC Genome Browser database: 2015 update. Nucleic Acids Res. 43(Database issue), D670-D681 (2015).

38. Consortium, E. P. et al. An integrated encyclopedia of DNA elements in the human genome. Nature 489, 57-74 (2012).

39. Khan, A. et al. JASPAR 2018: update of the open-access database of transcription factor binding profiles and its web framework. Nucleic Acids Res. 46(D1), D1284 (2018).

40. Curtis, C. et al. The genomic and transcriptomic architecture of 2,000 breast tumours reveals novel subgroups. Nature 486, 346-352 (2012).

41. Dvinge, $\mathrm{H}$. et al. The shaping and functional consequences of the microRNA landscape in breast cancer. Nature 497, 378-382 (2013).

42. Eisen, M. B., Spellman, P. T., Brown, P. O. \& Botstein, D. Cluster analysis and display of genome-wide expression patterns. Proc. Natl Acad. Sci. USA 95, 14863-14868 (1998).

43. Cerami, E. et al. The cBio cancer genomics portal: an open platform for exploring multidimensional cancer genomics data. Cancer Discov. 2, 401-404 (2012).

44. Shannon, P. et al. Cytoscape: a software environment for integrated models of biomolecular interaction networks. Genome Res. 13, 2498-2504 (2003).

45. Vakoc, C. R. et al. Proximity among distant regulatory elements at the beta-globin locus requires GATA-1 and FOG-1. Mol. Cell 17, 453-462 (2005).

46. Hagege, $\mathrm{H}$. et al. Quantitative analysis of chromosome conformation capture assays (3C-qPCR). Nat. Protoc. 2, 1722-1733 (2007)

47. Tan-Wong, S. M., French, J. D., Proudfoot, N. J. \& Brown, M. A. Dynamic interactions between the promoter and terminator regions of the mammalian BRCA1 gene. Proc. Natl Acad. Sci. USA 105, 5160-5165 (2008).

48. Bhan, A. et al. Antisense transcript long noncoding RNA (IncRNA) HOTAIR is transcriptionally induced by estradiol. J. Mol. Biol. 425, 3707-3722 (2013).

49. Milevskiy, M. J. et al. Long-range regulators of the IncRNA HOTAIR enhance its prognostic potential in breast cancer. Hum. Mol. Genet. 25, 3269-3283 (2016).

50. Ansari, K. I., Hussain, I., Shrestha, B., Kasiri, S. \& Mandal, S. S. HOXC6 Is transcriptionally regulated via coordination of MLL histone methylase and estrogen receptor in an estrogen environment. J. Mol. Biol. 411, 334-349 (2011).

51. Ansari, K. I., Hussain, I., Kasiri, S. \& Mandal, S. S. HOXC10 is overexpressed in breast cancer and transcriptionally regulated by estrogen via involvement of histone methylases MLL3 and MLL4. J. Mol. Endocrinol. 48, 61-75 (2012).

52. Ansari, K. I., Kasiri, S., Hussain, I. \& Mandal, S. S. Mixed lineage leukemia histone methylases play critical roles in estrogen-mediated regulation of HOXC13. FEBS J. 276, 7400-7411 (2009)

53. Watt, F. \& Molloy, P. L. Cytosine methylation prevents binding to DNA of a HeLa cell transcription factor required for optimal expression of the adenovirus major late promoter. Genes Dev. 2, 1136-1143 (1988).

54. Mai, T. et al. Estrogen receptors bind to and activate the HOXC4/HoxC4 promoter to potentiate HoxC4-mediated activation-induced cytosine deaminase induction, immunoglobulin class switch DNA recombination, and somatic hypermutation. J. Biol. Chem. 285, 37797-37810 (2010).

55. Patten, D. K. et al. Enhancer mapping uncovers phenotypic heterogeneity and evolution in patients with luminal breast cancer. Nat. Med. 24, 1469-1480 (2018).

56. Herz, H. M., Hu, D. \& Shilatifard, A. Enhancer malfunction in cancer. Mol. Cell 53, 859-866 (2014)

57. Heimberg, A. \& McGlinn, E. Building a robust a-p axis. Curr. Genom. 13, 278-288 (2012).

58. Sawado, T., Halow, J., Bender, M. A. \& Groudine, M. The beta-globin locus control region $(\mathrm{LCR})$ functions primarily by enhancing the transition from transcription initiation to elongation. Genes Dev. 17, 1009-1018 (2003).

59. Ko, J. Y., Oh, S. \& Yoo, K. H. Functional enhancers as master regulators of tissuespecific gene regulation and cancer development. Mol. Cells 40, 169-177 (2017).

60. Sotelo, J. et al. Long-range enhancers on 8q24 regulate c-Myc. Proc. Natl Acad. Sci. USA 107, 3001-3005 (2010).

61. Dostie, J. et al. Chromosome Conformation Capture Carbon Copy (5C): a massively parallel solution for mapping interactions between genomic elements. Genome Res. 16, 1299-1309 (2006).

62. Davies, J. O. et al. Multiplexed analysis of chromosome conformation at vastly improved sensitivity. Nat. Methods 13, 74-80 (2016). 
MicroRNA-196a is regulated by ER and is a prognostic biomarker in ER+... MJG Milevskiy et al.

632

63. Jiang, C. F. et al. Estrogen-induced miR-196a elevation promotes tumor growth and metastasis via targeting SPRED1 in breast cancer. Mol. Cancer 17, 83 (2018).

64. Conrad, T., Marsico, A., Gehre, M. \& Orom, U. A. Microprocessor activity controls differential miRNA biogenesis In Vivo. Cell Rep. 9, 542-554 (2014).

65. $\mathrm{Hu}, \mathrm{Z}$. et al. Common genetic variants in pre-microRNAs were associated with increased risk of breast cancer in Chinese women. Hum. Mutat. 30, 79-84 (2009).

66. Jedlinski, D. J., Gabrovska, P. N., Weinstein, S. R., Smith, R. A. \& Griffiths, L. R. Single nucleotide polymorphism in hsa-mir-196a-2 and breast cancer risk: a case control study. Twin. Res. Hum. Genet. 14, 417-421 (2011).

67. Siegfried, Z. et al. DNA methylation represses transcription in vivo. Nat. Genet. 22, 203-206 (1999).

68. Mohammed, H. et al. Endogenous purification reveals GREB1 as a key estrogen receptor regulatory factor. Cell Rep. 3, 342-349 (2013)

69. Hurtado, A., Holmes, K. A., Ross-Innes, C. S., Schmidt, D. \& Carroll, J. S. FOXA1 is a key determinant of estrogen receptor function and endocrine response. Nat. Genet. 43, 27-33 (2011).

70. Magnani, L., Ballantyne, E. B., Zhang, X. \& Lupien, M. PBX1 genomic pioneer function drives ERalpha signaling underlying progression in breast cancer. PLoS Genet. 7, e1002368 (2011).

71. Franco, H. L., Nagari, A. \& Kraus, W. L. TNFalpha signaling exposes latent estrogen receptor binding sites to alter the breast cancer cell transcriptome. Mol. Cell 58, 21-34 (2015).

72. Millour, J. et al. FOXM1 is a transcriptional target of ERalpha and has a critical role in breast cancer endocrine sensitivity and resistance. Oncogene 29, 2983-2995 (2010).

73. Kron, K. J., Bailey, S. D. \& Lupien, M. Enhancer alterations in cancer: a source for a cell identity crisis. Genome Med. 6, 77 (2014).

74. Chen, $H$. et al. A pan-cancer analysis of enhancer expression in nearly 9000 patient samples. Cell 173, 386-99 e12 (2018).
75. Whyte, W. A. et al. Master transcription factors and mediator establish superenhancers at key cell identity genes. Cell 153, 307-319 (2013).

76. $\mathrm{Hu}, \mathrm{Y}$. et al. Superenhancer reprogramming drives a B-cell-epithelial transition and high-risk leukemia. Genes Dev. 30, 1971-1990 (2016).

77. Burger, H. The menopausal transition-endocrinology. J. Sex. Med. 5, 2266-2273 (2008).

78. Hale, G. E., Robertson, D. M. \& Burger, H. G. The perimenopausal woman: endocrinology and management. J. Steroid Biochem. Mol. Biol. 142, 121-131 (2014).

79. Azim, H. A. Jr \& Partridge, A. H. Biology of breast cancer in young women. Breast Cancer Res. 16, 427 (2014).

80. Azim, H. A. Jr et al. Elucidating prognosis and biology of breast cancer arising in young women using gene expression profiling. Clin. Cancer Res. 18, 1341-1351 (2012).

(c) Open Access This article is licensed under a Creative Commons By Attribution 4.0 International License, which permits use, sharing, adaptation, distribution and reproduction in any medium or format, as long as you give appropriate credit to the original author(s) and the source, provide a link to the Creative Commons license, and indicate if changes were made. The images or other third party material in this article are included in the article's Creative Commons license, unless indicated otherwise in a credit line to the material. If material is not included in the article's Creative Commons license and your intended use is not permitted by statutory regulation or exceeds the permitted use, you will need to obtain permission directly from the copyright holder. To view a copy of this license, visit http://creativecommons. org/licenses/by/4.0/.

(c) The Author(s) 2019 